\title{
A Targets Prioritizing Method Based on Clustering Coefficient TSM
}

\author{
Shuna Cai ${ }^{1, a}$, Xiaomin Ran ${ }^{2, b}$ and Chao Wang ${ }^{3, c}$ \\ ${ }^{1}$ School of Shuna CAI., PLA Information Engineering University, Henan 450000, China \\ ${ }^{2}$ School of Xiaomin RAN., PLA Information Engineering University, Henan 450000, China \\ ${ }^{3}$ School of Chao Wang., PLA Information Engineering University, Henan 450000, China \\ a15981971760@163.com, brxmhkz@tom.com, cok135246sdu@163.com
}

Keywords: targets prioritizing, clustering coefficient, tactical significance map, analytic hierarchy process.

\begin{abstract}
An improved tactical significance map (CTSM) algorithm based clustering coefficient is proposed to deal with the multi-property targets prioritizing problem. The CTSM algorithm combines the target clustering coefficient with the traditional tactical significance map algorithm. The improved algorithm of the target priority is determined by three factors, target's own properties, relative attributes of target-region and target-target. The three factors are weighted by analytic hierarchy process. Simulation results show that the CTSM algorithm considers the problem more comprehensively and more in line with the practical application requirements.
\end{abstract}

\section{Introduction}

Target prioritizing is the basis of collaborative tracking, it is a comprehensive evaluation problem involves many domains.

So far, there are many research findings on target prioritizing, analytic hierarchy process (AHP) [1] , linear weighting method ${ }^{[2]}$ and fuzzy clustering ${ }^{[3]}$ and so on are the representative ones. Chen et al. ${ }^{[4]}$ Used the vague set for fuzzy decision for the first time. Tong Jun ${ }^{[5]}$ proposed the TOPSIS target prioritizing algorithm based on the vague set, but the above algorithm has great subjectivity and uncertainty for the fuzzy information measurement. The linear weighting method is the classical algorithm of target prioritizing and is widely used in multi-sensor management. However, the above fuzzy clustering method and linear weighting method ignore the non-linear relationship between target attribute and priority. The tactical significance map (TSM) proposed in [6] solves the nonlinear problem, but the algorithm does not consider the effect of targets' own properties on targets prioritizing. The weighted tactical significance map (WTSM) target prioritizing algorithm ${ }^{[7]}$ embodies the influence of the attribute on the target priority, and considers the nonlinear relationship between each attribute and the priority, but does not consider the relative trend between the targets.

In this paper, The improved algorithm CTSM proposed embodies the relative trend of the targets as one of the important factors, together with targets' own properties, the target-region trend constitute the objective function. The analytical hierarchy process is used to calculate the weights of three parts. Finally, through multi-set of simulation experiment proves the feasibility and effectiveness of the algorithm.

\section{Problem Description}

\subsection{Multi-Target Prioritizing Problem}

The application scenario is shown in Figure. 1 


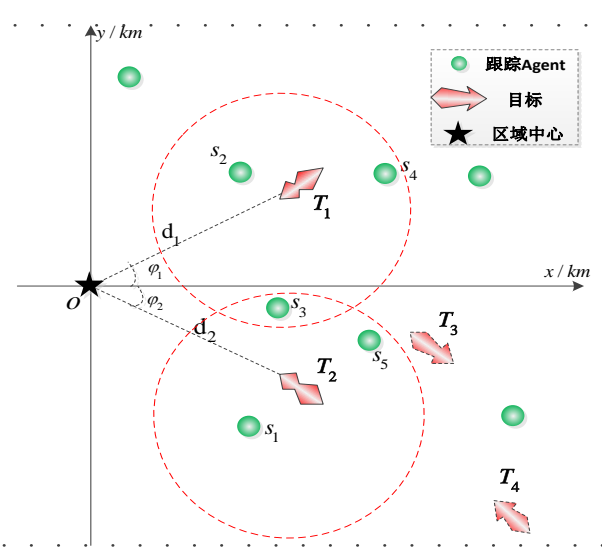

Figure 1. Application scenario

The rectangle is the monitoring area, and all the sensor platforms in the area constitute the monitoring and tracking system. The function of the tracking system is to identify, locate and track the objects that appear in the monitoring area. The target is randomly appear in the area, and the system needs to allocate reasonable tracking platforms for each target. If there are two or more targets in the monitoring area at a certain time, multiple targets may compete for one same tracking platform, but at the same time one tracking platform can only track one target. In Figure 1, target 1 and 2 compete at the same time, and the system must take a reasonable strategy to assign to one of the targets. Ranking the priority of the target is a more reasonable strategy, that is, the target that has high priority would get the platform.

\subsection{Factors Influencing Target Priorities}

There are many factors that determine the priority of the target. The previous studies mainly divide these factors into two categories: the target itself and the target-region relative trend. Based on the actual scene, this paper takes the factors of the relative trend between targets into account. The definition of these three influencing factors is given as follows.

Definition 1 target's own properties: refers to the targets' own type, the target attack range lie on physical structure, target type and athletic ability.

Definition 2 Target-region relative trend: refers to the relative trend between the target and the monitoring center, such as the distance from the center of the monitoring area, whether it is moving away from the center of the area, or is approaching the center of the area, what is the intention of the targets' invasion and so on.

Definition 3 The relative trend between targets: refers to the distribution state of the targets, including the degree of clustering between the targets, angle, distance and so on.

Since the targets prioritizing is a very complicated process, it is impossible to take all the factors into account. In this paper, six representative factors are selected as the factors, the target type ( T), the distance between targets and the center of the area(D), the angel between target moving direction and the center Angel(A), target velocity(V) and clustering coefficient (C). The target velocity, type, direction and type are identified by the initial measurements of the system. The target attack range can get from the target type database after recognizing the target type. The target clustering coefficient can get based the target distance and distribution.

\section{Targets Prioritizing Based on CTSM}

\subsection{Tactical Significance Map Algorithm}

The objective function of the tactical significance map algorithm takes three influencing factors into account, target velocity, moving direction and distance. The target state vector is $\vec{x}_{i}=\left[\vec{p}_{i}, \vec{v}_{i}\right]=\left[p_{x, i}, p_{y, i}, \mathrm{v}_{x, i}, v_{y, i}\right]$, where $\vec{p}_{i}$ is the position vector, $\vec{v}_{i}$ is the velocity vector, $\vec{x}_{0}=\left[p_{x, 0}, p_{y, 0}\right]=[0,0]$ is the coordinate of the origin $\mathrm{O}$, the origin $\mathrm{O}$ is the monitoring center, and the target-region distance can be expressed as follows 


$$
d_{i}=\sqrt{\left(p_{x, i}-p_{x, 0}\right)^{2}+\left(p_{y, i}-p_{y, 0}\right)^{2}}
$$

The moving direction is the angle between the position vector and the velocity vector, denoted by $\theta\left(\vec{p}_{i}, \vec{v}_{i}\right)$, which is derived from the follow formula

$$
\theta\left(\vec{p}_{i}, \vec{v}_{i}\right)=\cos ^{-1}\left(\frac{\left(\vec{p}_{i}, \vec{v}_{i}\right)}{\left\|\vec{p}_{i}\right\|\left\|\vec{v}_{i}\right\|}\right)
$$

\subsection{CTSM Based Targets Prioritizing Algorithm}

British well-known engineer Lanchester ${ }^{[8]}$ proved that in modern war conditions, the strategy concentrating forces then attacking the enemy separately, can effectively improve the efficiency of the battle. The proposed CTSM target prioritizing method just take full use of the thinking.

The target priority should be in a negative relationship with the target clustering coefficient, that is, when the other parameters are the same, the system preferentially selects the relative isolated target. The relationship between the target clustering coefficient and the target priority function can be expressed as follows

$$
f_{T S M}\left(\delta_{i}\right) \sim \exp \left(-\delta_{i}^{2}\right)
$$

The target clustering coefficient represents the relative trend between the targets. The algorithm establishes three priority sub-functions, according to the three category factors respectively. They are add with corresponding weights. The priority function expression as follows

$$
\begin{aligned}
& f_{\text {GWTSM }}\left(\vec{x}_{i}, \xi_{i}, \beta_{i}, \delta_{i}\right)=\varphi_{1} f_{T P}\left(\xi_{i}, \beta_{i}\right)+\varphi_{2} f_{R S}\left(\vec{x}_{i}\right)+\varphi_{3} f_{3}\left(\delta_{i}\right) \\
& =\varphi_{1} \cdot \exp \left(\frac{-\xi_{i}^{2}}{2 \beta_{i}^{2}}\right)+\varphi_{2} \cdot \exp \left(\frac{-d_{i}^{2}}{2\left(1-\frac{\theta\left(\vec{p}_{i}, \vec{v}_{i}\right)}{\pi}\right)^{2}\left(k_{0}\left\|\vec{v}_{i}\right\|+m_{0}\right)^{2}}\right)+\varphi_{3} \cdot \exp \left(-6 \delta_{i}^{2}\right)
\end{aligned}
$$

Where, $\xi_{i}=d_{i}-l_{i}$ is the difference between target to region center distance and target attack range, $\varphi_{1}, \varphi_{2}$ and $\varphi_{3}$ respectively are the weights of target own properties, the target-region trend and the relative trend of target-target.

\section{Simulation Experiment and Result Analysis}

\subsection{Target Type Scoring Method}

Obviously, for the target prioritizing, it is only necessary to know the order of the priority function values other than necessary to know the absolute value of the objective function. In order to facilitate the analysis of the performance of the algorithm, according to the general hierarchy analysis, the target is divided into five levels: dangerous, more dangerous, generally dangerous, very dangerous and more than very dangerous, respectively, the corresponding score is $1,2,3,4,5$, as shown in Table 1.

Table 1. Target Type Scoring Category

\begin{tabular}{cccccc}
\hline Dangerous degree & Danger & More dangerous & General danger & Very dangerous & Very dangerous \\
\hline score & 1 & 2 & 3 & 4 & 5 \\
\hline
\end{tabular}

\subsection{Measurement of The Clustering Coefficient}

In this paper, the artificial potential model is used to measure the clustering coefficient []. In the so-called artificial potential model, the concept of "potential field" in physics is used to construct the battlefield situation analysis model based on the potential strength. In the model, each of the targets are saw as a "positive charge" in the "potential field". The total "potential strength" of a target is the sum of the potential strengths of the other targets at the target, and the larger the sum, the greater the degree of clustering. In order to facilitate the application of the model, the monitoring area is divided into a number of squares, the cells where the targets located the potential strength is " +1 ", the potential strength to the neighboring cells gradually attenuating. In this paper, the attenuation coefficient take a multiple of 0.5 , that is, the potential value will increase 0.5 in the adjacent cells just because the 
target. and 0.25 value increase in the sub-adjacent cell, and analogous for all units . Then, the total "potential strengths" value of each cell stem from all other cells can be got.

Figure 2 and Figure 3 show the potential diffusion results of the two target agents, and Figure 4 shows the superposition results of the two target diffusion potentials.

\begin{tabular}{|c|c|c|c|c|}
\hline 0.125 & & 0.25 & 0.25 & 0.25 \\
\hline 0.125 & 0.25 & 0.5 & 0.5 & 0.5 \\
\hline 0.125 & 0.2 & 0.5 & +1 & 0.5 \\
\hline 0.125 & 0.25 & 0.5 & 0.5 & 0.5 \\
\hline 0.125 & 0.25 & 0.25 & 0.25 & 0.25 \\
\hline
\end{tabular}

\begin{tabular}{|l|l|l|l|l|}
\hline 0.0625 & 0.0625 & 0.0625 & 0.0625 & 0.0625 \\
\hline 0.125 & 0.125 & 0.125 & 0.125 & 0.0625 \\
\cline { 2 - 5 } & & & & \\
\hline 0.25 & 0.25 & 0.25 & 0.125 & 0.0625 \\
\hline 0.5 & 0.5 & 0.25 & 0.125 & 0.0625 \\
\hline+1 & 0.5 & 0.25 & 0.125 & 0.0625 \\
\hline
\end{tabular}

Fig. 2 potential diffusion results of target 1 Fig. 3 potential diffusion results of target 2

\begin{tabular}{|c|c|c|c|c|}
\hline 0.1875 & 0.3125 & 0.3125 & 0.3125 & 0.3125 \\
\hline 0.25 & 0.375 & 0.625 & 0.625 & 0.5625 \\
\hline 0.375 & 0.5 & 0.75 & 1.125 & 0.5625 \\
\hline 0.625 & 0.75 & 0.75 & 0.625 & 0.5625 \\
\hline 1.125 & 0.75 & 0.5 & 0.375 & 0.3125 \\
\hline
\end{tabular}

Fig. 4 compound potential

\subsection{Comparison With TSM Algorithm}

The global parameters are set as follows: $d=5 \mathrm{~km}, l=1 \mathrm{~km}, \theta=0$. The parameters for TSM algorithm: $k_{0}=100, \mathrm{~m}_{0}=1200, k_{1}=2 \times 10^{6}, k_{2}=6$. The parameters for CTSM: $k_{0}=100$, $\mathrm{m}_{0}=1200$. The relationship about the target priority, velocity and the clustering coefficient between the two algorithms are shown in Figure 5 and 6.

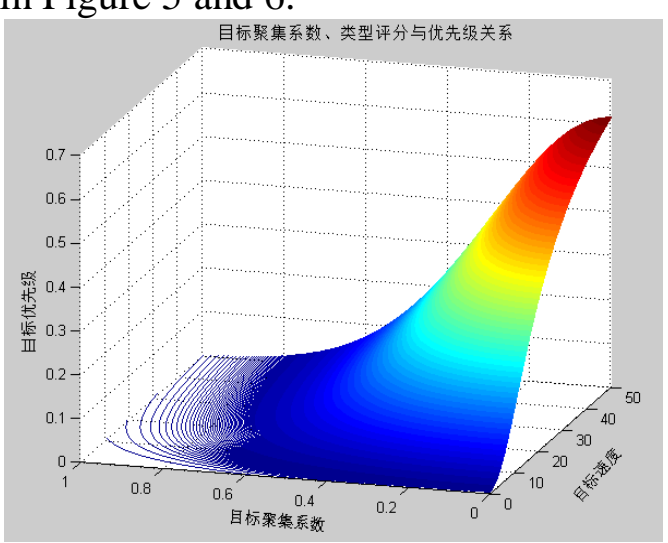

Fig. 5 TSM algorithm simulation result 


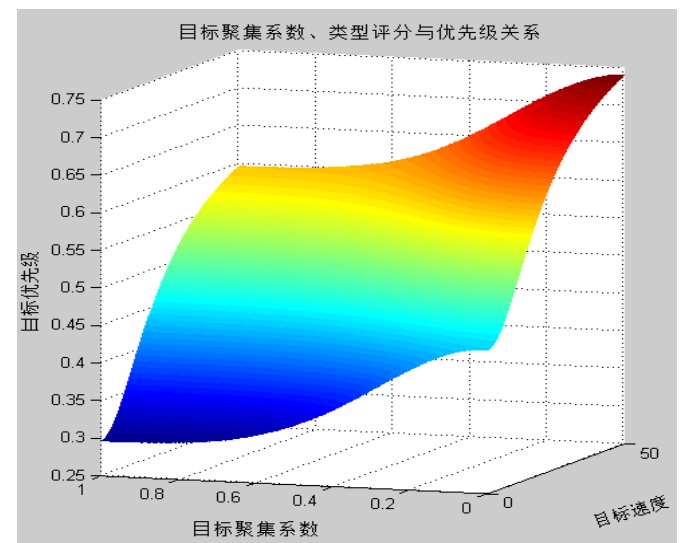

Fig. 6 CTSM algorithm simulation result

It can be seen from the simulation results that in the TSM algorithm, when the target clustering coefficient is large, the rate of the target priority $(\mathrm{Pr})$ increases with the velocity is always zero, but the rate is too large when the clustering coefficient is small. While in the CTSM algorithm simulation, it can be seen that the target priority increases steadily with the target clustering coefficient, and the conclusion of the simulations for priority with velocity and distance is similar.

The specific comparison simulation is as follows: as in the figure 7 and 8 , the coordinate of the center is $(100,100)$.There are three targets respectively located at $(110,90),(135,140)$ and $(135,60)$ competing for the one tracking platform $\mathrm{P}$, and their velocity respectively is $25 \mathrm{~km} / \mathrm{h}$, $20 \mathrm{~km} / \mathrm{h}$ and $25 \mathrm{~km} / \mathrm{h}$, and the moving direction angles respectively are $\pi, 0$ and 0 , and their $\beta$ all are 4. According to the TSM algorithm, the sorting results of the three targets are shown in Figure 1, and the sorting results according to the CTSM algorithm are shown in Figure 2. It can be seen from the simulation results that the CTSM algorithm is more reasonable because the target 1 is moving closer to the center point, but it is moving in the opposite direction to the center, so its priority should be the minimum, the velocity of target 2 is slightly smaller than the target 3 , but its clustering coefficient is much smaller than the target 3 , with comprehensive assessment of the factors, the priority of target 2 should be the highest. The CTSM algorithm not only reflects the influence of the target's own attributes and the target-region relative trend on the priority, but also considers the important influencing of the relative trend between the targets, which is more in line with the actual needs of the battlefield.

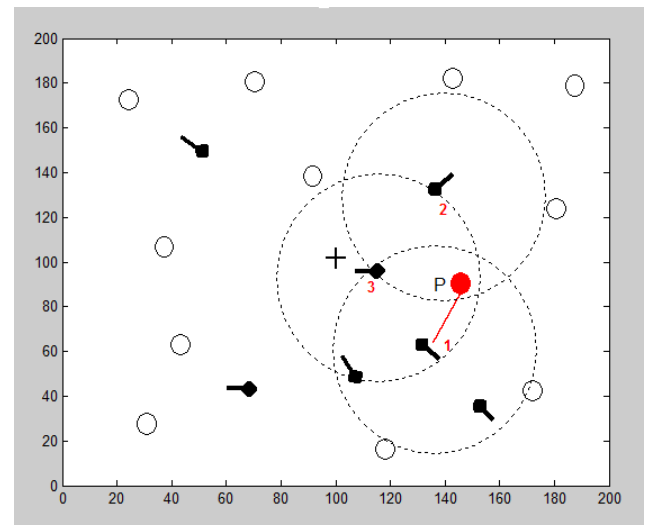

Fig. 7 The performance of TSM algorithm 


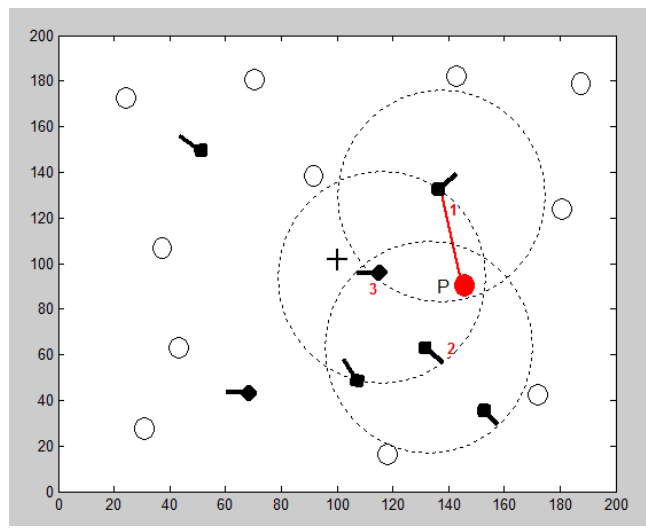

Fig. 8 The performance of CTSM algorithm

\section{Conclusion}

An improved algorithm CTSM for target prioritizing is proposed. The algorithm combines the target clustering coefficient with the tactical significance map according to the Lanchester equation. The sub-functions are weighted by analytic hierarchy process. The simulation results show that the proposed algorithm is more comprehensive, which reflects the non-linear relationship between the factors and the priority, and avoids the situation that one factor controls the global result .It satisfies the requirements of modern battlefield.

\section{References}

[1]. Wang L F, Xu S B. An Introduction to Analytic Hierarchy Process [M].Beijing: Renmin University of China press, 1990, p.201-203.

[2]. Liu X S. Research on Sensor Management Method [D]. Xi'an: Northwestern Polytechnic University, 2000, p.102-103.

[3]. Tan P, Cao P. Ground Subsidence Prediction Based on Gray Correlation Support Vector Machine [J]. Journal of Central South University, 2012, 43 (2):229-234.

[4]. Chen S M, Tan J M. Handling Multi-criteria Fuzzy Decision-making Problems Based on Vague Set Theory [J]. Fuzzy Sets \& Systems, 1994, 67 (2):163-172.

[5]. Tong J, Shan G L. Solving Target Priority by TOPSIS Method Based on Vague Set [J]. Fire Control \& Command Control, 2012, 37 (5):140-143.

[6]. El-Fallah A, Zatcaalo A, et al. Unified Bayesian Situation Assessment Sensor Management [C].Proc. of SPIE Signal Processing, Sensor Fusion, and Target Recognition, 2005, p.253-264.

[7]. Wei S Y, Zhang J, Ran X M, etc. A Multi-objective Prioritizing Method Based on Weighted TSM [J]. Journal of Information Engineering University, 2015, 16 (3), p.292-297.

[8]. Li Z Q. Research of Information Warfare Modeling and Simulation Based on Complex System Theory [D]. National Defense University, 2006, p.136-137. 